\title{
Talleres de capacitación de convivencia y buen trato para educadores de niños con necesidades educativas especiales
}

\section{$M^{a}$ Teresa Muñoz Quezada ${ }^{1}$, Boris Lucero Mondaca $^{2}$}

\author{
${ }^{1}$ Departamento de Psicología, Universidad Católica del Maule, \\ Ciudad de Talca, Chile. \\ ${ }^{2}$ Psicólogo del Hogar de Menores Belén y Fundación CRATE \\ de la Ciudad de Talca, Chile.
}

\section{Chile}

mtmunoz@ucm.cl 


\section{Resumen}

La siguiente experiencia describe el proceso de implementación y los resultados de los talleres que se realizan desde el año 2003 con las educadoras del hogar de menores con discapacidad Belén de Talca. Los talleres consisten en una capacitación constante con el personal sobre la convivencia y el buen trato, con el fin de generar una mejor relación interpersonal dentro del establecimiento, una intervención eficaz, asertiva y oportuna con los residentes y generar estrategias de autocuidado. La metodología de los talleres se enfoca a partir de grupos de trabajo, con experiencias teóricas y aplicadas. Las experiencias aplicadas durante los tres años, han dado como resultado que las educadoras además de promover el buen trato, logren desarrollar habilidades profesionales y estrategias de acción que mejoran el clima y las relaciones humanas dentro del hogar, observándose a partir de ello mayores conductas adaptativas en los niños y jóvenes residentes.

Palabras Clave: Buen trato, educadores, discapacidad

Recepción del artículo: 01-09-2006

Aceptación provisional: $\quad 26-02-2007$

Aceptación definitiva: $\quad$ 18-05-2007 


\section{Introducción}

El buen trato ha sido un tema que se viene desarrollando transversalmente en los diversos contextos políticos, sociales y educacionales de nuestro país desde la transición a la democracia. La temática es abordada en los objetivos fundamentales en educación, principalmente referidos a los objetivos fundamentales transversales, incluyendo en el proceso de enseñanza - aprendizaje el respeto por los derechos humanos, promoviendo en los estudiantes el sentido ético y respeto por la vida, el desarrollo personal y la participación y compromiso social (Magendzo, Donoso y Rodas, 1997, MINEDUC, 2004).

En el contexto de salud también se incluye la propuesta del desarrollo del buen trato, relacionado principalmente con la atención del personal de salud a las personas que se atienden en centros y hospitales tanto del sector público como privado, espacios donde se promueven los derechos del paciente a recibir una atención digna y de calidad. El Servicio Nacional de la Mujer (SERNAM), el Ministerio de Salud (MINSAL) y el Servicio Nacional de Menores (SENAME), se convierten en Chile en entidades públicas que promulgan el derecho a un buen trato para todas las personas, sin discriminar condición ni etnia, promoviendo el desarrollo de relaciones humanas respetuosas y cálidas dentro de los centros de atención a la comunidad, tanto a nivel de los funcionarios, como de las personas que reciben atención en dichas instituciones, con el fin de promoverlo en sus diversos programas al contexto social en general.

En este sentido el SENAME (2006) ha generado como parte de los programas de atención a los niños, niñas y jóvenes, la promoción del buen trato. Para esto se han llevado a cabo una serie de jornadas y capacitaciones con el fin de potenciar la comunicación, habilidades sociales, la asertividad, la expresión de afectos, como claves para intervenir con los niños y sus familias, y así disminuir las instancias de violencia y negligencia hacia los menores, además de promover una calidad de vida más sana y constructiva para el desarrollo integral de la persona.

Se entiende como buen trato una actitud, una forma de vida, y una toma de conciencia que involucra el conocimiento personal y la aceptación del otro como legítimo otro, dejando entre paréntesis nuestros prejuicios, y que nos permite desarrollar un vínculo y una relación interpersonal efectiva, carente de amenaza, plena en la integración y apertura consigo mismo 
y con los demás (Aron y Milicic, 1993; Barudy, 2003; Maturana y Verden - Zöller; 2000; Rogers, 2000; Montes y Montoya, 2003).

Según Montes y Montoya (2003) el buen trato es una instancia de comunicación y relación humana recíproca, considerándose como un encuentro, que se relaciona con la manera en que se percibe a los demás y cómo se percibe a sí mismo. Se considera como un proceso social de aprendizajes y cambio cultural entre los agentes socializadores de los niños, niñas y jóvenes. Por lo tanto, para poder promover el buen trato, se requiere hacer frente a la cultura del maltrato, que valida la violencia como un elemento de intervención. El maltrato al ser aprendido se puede modificar, y generar a cambio elementos culturales que promuevan la afectividad y relaciones interpersonales positivas en el contexto de formación e interacción cotidiana entre niños y adultos.

Considerando que los distintos ambientes donde los niños interactúan son claves en su desarrollo psicológico, físico, social y espiritual (MINSAL, 1999, MINEDUC, 2004), se debe tener presente en las intervenciones realizadas por las educadoras, las relaciones interpersonales y comunicación que se dan en el centro de atención, la escuela y entre los pares, para la prevención de conductas agresivas (De la Fuente, Peralta y Sánchez, 2006; Justicia et al, 2006). Aquí no solo aprenden a conformar su identidad personal y social, sino también empiezan a generar los vínculos y relaciones sociales con otros.

Algunos estudios (Aracena, et al., 2000; Marty y Carvajal, 2005; Robaina, 2001; Vizcarra, Cortés, Bustos, Alarcón y Muñoz, 2001,) muestran que existe relación entre tener una visión negativa de los niños y el uso de castigo físico por parte de los adultos, lo que está asociado al hecho de que muchas veces las expectativas que tienen los padres y adultos hacia los niños no corresponden con las capacidades y la habilidad de comprensión que ellos poseen (principalmente en cuanto al nivel de desarrollo cognitivo y socioafectivo limitado y propio para su edad).

Barudy (2003) propone un modelo de intervención alternativo a la violencia para promover el buen trato. Este modelo parte de la biología del amor, donde se considera a las personas como lo más importante. Esta visión genera la co-construcción de los modelos de relaciones interpersonales, familiares, profesionales y sociales, que genera una relación altruista, personas defensoras de los derechos de las personas y del respeto de la naturaleza. Este autor 
menciona que existen muchas intervenciones de protección perjudiciales para niños y niñas producto de incompetencia de los profesionales que supuestamente les protegen. Considera que es importante generar redes entre los profesionales para mejorar sus competencias, prevenir el estrés laboral de quienes atienden a niños en riesgo psicosocial, y evitar los etiquetamientos y desensibilización del sufrimiento y problemática de los niños, niñas y jóvenes.

Por otro lado, es importante generar instancias de capacitación a los profesionales que atienden a los menores en riesgo psicosocial, considerando el alto nivel de estrés que genera dicha condición en los equipos que los atienden. Diversos estudios (Morales, Pérez y Menares, 2003; Montgomery y Rupp, 2005; Quintana, 2005) muestran que se produce desgaste emocional en la atención de personas que vivencian situaciones de riesgo psicosocial y maltrato, generando riesgo para la salud mental, afectando su compromiso emocional con el trabajo, y por ende, influyendo en la entrega de un servicio poco eficiente y despersonalizado hacia los menores, quienes, al percibir esta situación generan más conductas desadaptativas y de desamparo, produciendo así una cadena de situaciones estresantes.

A partir de lo anterior, surge el interés por parte de la institución de perfeccionar a las educadoras de trato directo. El objetivo general de los talleres consiste en capacitar a las educadoras en convivencia y buen trato, con el fin de que lo apliquen en la intervención con los residentes de la institución.

\section{Método}

\section{Participantes}

El hogar Belén de Talca es un centro de protección simple con internado para niños, niñas y jóvenes con discapacidad leve, moderada, severa y profunda. También atiende a adultos, debido a que el egreso de los beneficiarios es prácticamente inexistente, debido a que por lo general son abandonados, se manifiesta poco interés de parte de sus familias de origen, o sus familias viven en condiciones de pobreza extrema que no les permite brindar los cuidados y atención que los jóvenes necesitan. La población de personas que se atienden en el hogar corresponde a 32, donde 29 son menores de 24 años. Estos últimos reciben subvención de SENAME. Además presentan diversas discapacidades, comprendidas en retraso mental, trastornos psiquiátricos, discapacidad auditiva, trastornos de la comunicación, parálisis cerebral y trastornos del desarrollo. Las edades de los beneficiarios fluctúan entre los 4 y 40 años, quie- 
nes están en riesgo psicosocial y abandono, en términos de pobreza, negligencia, maltrato infantil y violencia intrafamiliar, y generalmente provienen de zonas rurales, alrededor de la comuna de Talca.

La muestra está conformada por la cantidad de educadores de trato directo, incluida la directora del centro, que corresponden a 12 mujeres cuyas edades fluctúan entre los 24 y 55 años.

\section{Instrumentos}

Para recoger los datos se utilizaron entrevistas individuales y diversas técnicas tales como: lluvia de ideas, construcción del árbol de problemas/soluciones, y técnica Delphi. Además se utilizo el Inventario de Burnout de Maslach para medición de estrés laboral y la Escala de estrés psicosocial de Holmes y Rahe.

La escala Maslach (Burnout Inventory, MBI-HS) es un instrumento que ha sido validado y es el de mayor uso en la evaluación y medición de la presencia del síndrome de Burnout. El cuestionario consta de 22 ítems distribuidos en tres factores: (1) agotamiento emocional (CE), (2) despersonalización (DP), y (3) falta de realización personal y en el trabajo (RP). A partir de estos tres factores se construyen a su vez las tres subescalas del MBI, en donde se suman los ítems de acuerdo con la dimensión, las dos primeras son positivas y la última se considera negativa. En dicho sentido, un sujeto que se encuentra en la categoría "alto" en las dimensiones de agotamiento emocional y despersonalización, y "bajo" para realización personal en el trabajo puede ser categorizado como un sujeto con altos sentimientos de estar "quemado". Esta escala fue validada en Chile por Buzzetti (2005) en educadores, y se correlaciona con la confiabilidad y validez del instrumento original obteniendo una alta consitencia interna (entre 0.80 y 0.90$)$.

Por su parte, la escala de estrés psicosocial de Holmes y Rahe (1967 )y denominada "Escala de Reajuste Social", SRRS), consiste en una enumeración de los 43 sucesos estresantes más frecuentes y relevantes que se presentan en la vida de las personas de acuerdo a lo observado en historias clínicas, denominados "acontecimientos vitales ("life events")". Al momento de contestar la escala se le debe otorgar a cada uno de ellos una determinada puntuación en una escala porcentual, donde sobre los 250 puntos corresponde a una situación de "sobre estrés". Una validación del instrumento fue aplicada por Rivera, Vollmer, Aravena y 
Carmona (1985) en una muestra de estudiantes universitarios y funcionarios administrativos. Aquí se presentó una correlación significativa $(\mathrm{r}=0.85, \mathrm{p}<0.001)$ con los resultados presentados por los autores originales del test en una muestra de norteamericanos.

\section{Procedimiento}

\section{Diseño de la intervención y diagnóstico inicial}

Los talleres se realizan con las educadoras desde el año 2003. La metodología utilizada es de la modalidad de grupos de trabajo participativo, con experiencias teóricas y aplicadas, en donde no sólo se tratan contenidos sobre la temática, sino que también se evalúa en terreno, las instancias de intervención que se realizan con los menores y jóvenes. Se consideró oportuno utilizar dicha metodología con el fin de generar un espacio de co-construcción de conocimientos y experiencias más atingentes a la realidad que vivencian en el día a día las educadoras con los niños y jóvenes.

Las intervenciones grupales están orientadas a mejorar y potenciar el buen trato en el centro de atención, entre el personal y desde el personal hacia los niños (as) y jóvenes. Para esto se realiza un diagnóstico inicial cada año para generar un árbol de problemas y soluciones desde las educadoras en conjunto con los psicólogos, con el fin de priorizar las temáticas que requieren de una rápida intervención y son esenciales para mejorar el trato del personal a los niños y jóvenes. La información se recopiló a partir de entrevistas individuales con la directora y entrevistas focales con el grupo de educadoras, donde se aplicaron las técnicas e instrumentos mencionados.

A partir de los resultados de estos diagnósticos, se observa que las principales dificultades que identifican las educadoras serían el estrés al cual se ven expuestas tanto producto de la carga de trabajo como de eventos personales y familiares que afectan su calidad de vida y salud mental, lo que también estaría vinculado a la falta de estrategias de autocuidado y afrontamiento al estrés. Durante el año 2005 y 2006 obtuvieron en promedio un nivel medio de estrés laboral y un nivel alto de estrés psicosocial, donde el estrés psicosocial se relacionaría con el cansancio emocional que genera la jornada laboral. Ellas explican que el estrés provocaría ciertas situaciones de conflicto entre las educadoras, y por otro lado, agotamiento frente al trabajo, lo que podría derivar en que el servicio que brindan a los menores no sea en ocasiones tan eficiente. El temor principal del equipo es llegar a enfermar física y mentalmente y 
no poder intervenir con los niños y niñas de manera cálida y eficaz. Para esto se propone como solución realizar talleres de buen trato y autocuidado, y talleres de relajación.

Otro elemento que consideran importante es el olvido de las acciones informadas y comprometidas entre los miembros del equipo de educadoras con respecto a las labores de rutina, lo que en ciertas oportunidades genera una atención poco eficiente en el cuidado de los niños. Como solución proponen buscar estrategias para recordar y potenciar la gestión de los equipos de trabajo. Para eso realizan un taller de mejoramiento de la gestión y trabajo de equipo.

También se reconoce la dificultad en la comunicación tanto entre las personas pertenecientes al equipo de educadoras, como con las residentes del hogar (especialmente con las jóvenes y adultas). Referente a lo anterior, resulta especialmente crítico la existencia de comentarios mal intencionados dentro del equipo de educadoras, los cuales no se explicitan en una comunicación transparente y clara dirigida hacia mejorar el cumplimiento de los compromisos laborales del equipo, sino más bien generan una cultura del rumor, llevando a un latente enrarecimiento del clima laboral, entrabando con esto un funcionamiento sinérgico y fluido del equipo. Frente al punto anterior, las educadoras plantean como solución generar una comunicación más abierta y directa, por lo cual se propone realizar un taller de comunicación y asertividad, con el fin de mejorar las relaciones humanas, la confianza y la calidad en la entrega de la intervención con los menores, jóvenes y adultos.

Otro elemento importante es lo que se refiere a su dificultad con el manejo conductual, que consideran clave para poder enseñar el buen trato y aplicarlo entre los niños. Frente a esto se proponen diversas soluciones: (1) Realizar reuniones clínicas semanales donde puedan discutir con el psicólogo las posibles soluciones del caso; (2) desarrollar un taller de capacitación donde comprendan las características de las etapas de desarrollo psicológico de los niños, niñas y jóvenes según las discapacidades y situación social que presentan; (3) realizar un taller de buen trato en la atención de los niños (as), jóvenes y adultos y (4) efectuar un taller de manejo conductual.

\section{Implementacion de las actividades e intervención}

A continuación se describen las temáticas abordadas y por desarrollar según el año de aplicación: 
Año 2003:

- Conocer las características y desarrollo de la población con necesidades educativas especiales y las estrategias de intervención apropiadas: Se entregó formación a las educadoras para el conocimiento y comprensión de las discapacidades con el fin de que pudieran adecuar la intervención, considerando además los problemas psicosociales y los diferentes estados evolutivos.

- Desarrollar un buen trato en la atención con niños, niñas, jóvenes y adultos con discapacidad: Consistió en un taller que promovía la capacitación de las educadoras en estrategias y técnicas de intervención y atención desde el modelo de buen trato para los niños (as), jóvenes y adultos en situación de abandono y con discapacidad.

Año 2004:

- El buen trato y manejo conductual: Consistió en reuniones semanales en donde se trabajaban con las educadoras el aprendizaje y aplicación de técnicas de manejo conductual orientadas desde el modelo de buen trato, considerando la discapacidad y etapa de desarrollo de los beneficiarios.

- Técnicas de relajación para el manejo conductual: Consistió específicamente en el aprendizaje y aplicación de técnicas de relajación para el control y manejo conductual de los niños, niñas, jóvenes y adultos en situaciones de descontrol emocional y de comportamientos desadaptados.

Año 2005:

- Taller para mejorar la gestión de las educadoras en la atención de los niños y jóvenes del hogar: Pretendía potenciar la gestión de los equipo de trabajo de las educadoras, con el fin de brindar una atención de calidad, además de mejorar las relaciones interpersonales entre las educadoras.

- Técnicas de relajación para el personal: Consistió en el aprendizaje y aplicación de técnicas de relajación mental y corporal con el fin de enfrentar los niveles de estrés del equipo de trabajo.

Año 2006:

- Taller de autocuidado y relaciones interpersonales en el trabajo: Consistió en el aprendizaje y aplicación de estrategias y técnicas de autocuidado como desarrollo de la autoestima, autoexploración de pensamientos y sentimientos frente a las situaciones 
complejas, estrategias de autocontrol y modificación cognitiva de pensamientos negativos, estrategias y técnicas de afrontamiento al estrés y estrategias de asertividad y promoción de relaciones de buen trato entre las educadoras.

- Taller de comunicación efectiva para el buen trato (por realizar a partir de agosto): Pretende capacitar y fortalecer en las educadoras el uso de una comunicación efectiva, donde se promueva el escuchar activo, responder compresivo y expresar propositito con el fin de fortalecer conductas de buen trato tanto entre las educadoras como en la atención con los beneficiarios.

\section{Evaluacion de las actividades}

Para evaluar las actividades se utilizaron cuestionarios y entrevistas, donde se incluían ítems de puntuación tipo escala likert, y también ítems tanto de preguntas cerradas como abiertas. A su vez, se incluyeron entrevistas de focus group con el equipo de educadoras, para conocer y observar qué cambios se habían evidenciado en sus áreas de desempeño (por ejemplo en el manejo conductual). En la construcción de los cuestionarios se consideraron elementos que habían surgido desde la actividad inicial de diagnóstico, siendo posible a partir de ello incluir los objetivos para los cuales las actividades e intervenciones habían sido diseñadas. A traves de estos instrumentos, las educadoras participantes brindaron retroalimentación con respecto a los resultados que habían observado desde el desarrollo de las actividades y la intervención.

Finalmente, tambien se obtuvo un referente evaluativo adicional a partir de la aplicación en años consecutivos de los instrumentos estandarizados que se utilizaron para medir estrés laboral y psicosocial.

\section{Resultados}

A continuación se presentarán los resultados de las evaluaciones realizadas con respecto a las experiencias de los talleres. Primero se presentarán los resultados cualitativos que emergieron de los grupos focales, las entrevistas cuestionarios, y posteriormente los datos más cuantitativos.

Con respecto a los datos cualitativos, se observa que las experiencias aplicadas en los tres años consecutivos, han permitido que las educadoras no sólo lograran promover el buen trato en la atención de los niños, niñas, jóvenes y adultos, sino también lo han considerado 
como parte de un estilo de vida, que potencia las relaciones humanas y permite una mejor calidad de vida, esto se observa en los siguientes comentarios:

"Los talleres nos han permitido mejorar la relación con los niños y también en nuestras familias, yo aplico los ejercicios en mi casa, con el grupo de niños, a mi hija, y me han resultado/.../uno está más tranquila, pese al sacrificio, se duerme mejor, se pueden hacer las cosas más concentrada"

"Se podría continuar trabajando esto con nosotras, nos falta, pero lo que hemos aprendido ha servido, sobretodo los ejercicios de autocuidado, de comunicación, aunque todavía nos falta trabajar más entre nosotras".

Ahora se es más conciente de lo que uno piensa y dice, hay que cuidar más no sólo las palabras sino el cómo se dice, en todas partes, en la casa, además del trabajo..."

"Es parte de la rutina del hogar, ahora se trabaja autocuidado con los niños, en horarios después del estudio, o el fin de semana a media tarde o en la mañana/.../también las chiquillas aprendieron los ejercicios y se los aplican entre ellas o con nosotras".

También, las participantes mencionan que se perciben más asertivas en sus intervenciones, aplicando las estrategias de comunicación personalizada en la atención con los beneficiarios y también en su vida cotidiana. Consideran que se sienten con más herramientas de formación para intervenir como mediadoras en los diversos conflictos que se presentan entre los niños y jóvenes, y promover una convivencia más sana y humana entre ellos y las educadoras, además de percibir que al saber más sobre las características de sus discapacidades y desarrollo psicológico, les ha permitido comprender los diversos procesos y formas de ser que presentan en su interacción cotidiana:

"Hay más cambios positivos, los niños obedecen o más que eso, entienden lo que uno les quiere decir, es que también me pongo en el lugar de él, sé que por su discapacidad tiene que ser más concreta la explicación o el llamado de atención”.

"Los talleres nos permitieron saber comunicarnos y entender a los niños y jóvenes según su discapacidad, etapa de vida e historia personal, todos son distintos, por lo tanto tienen respuestas diferentes, nadie es igual, por lo mismo requieren diferentes tratos, explicaciones, intervenciones/.../también descubrimos que nosotras somos distintas".

Por otro lado, se ha logrado generar un clima de relaciones humanas constructivo y nutritivo dentro del hogar, donde se promueve el diálogo, se considera la opinión del otro, generando un espacio de respeto y aceptación mutua, donde se confrontan las situaciones de 
conflicto considerando la integridad y características de los menores, además de potenciarse las relaciones interpersonales entre el personal, donde se reconoce que han mejorado, aunque aún se requiere trabajar más la asertividad y la comunicación personalizada:

"Ahora sabemos mediar en los conflictos, identificarlos, aunque todavía nos falta, pero estamos mucho mejor, podemos decirnos las cosas sin llegar a gritar”.

"Los niños están más tranquilos si nos ven tranquilas a nosotras"

"Podemos ayudarlos a resolver mejor sus problemas, aplicando el escuchar activo, respoder comprensivo y expresar de manera constructiva, bien concreto, para que nos entendamos"

“Ahora no están tan violentos, creo que en esto ha influido no sólo los tratamientos médicos, sino también la estrategia con la cual resolvemos los problemas y los ejercicios de relajación, además hay más comunicación, se da más espacio a escuchar”

Las educadoras manifiestan la necesidad de seguir implementando estos talleres, quienes además de percibirlo como una herramienta de formación continua en su labor con la población que atienden, también lo perciben como un espacio de reflexión y aprendizaje tanto de sí mismas como de las relaciones interpersonales. Manifiestan, además, la importancia de la supervisión constante de parte del psicólogo, como una forma de analizar las creencias personales, analizar los casos clínicos, mejorar las estrategias de manejo conductual y potenciar las prácticas educativas, además de generarse un espacio de apoyo y retroalimentación de sus propios aprendizajes e intervenciones:

"Todavía tenemos que superarnos, igual hay problemas entre nosotras que tenemos que conversar, a veces las cosas no se hacen bien o se hacen a medias, o a alguien se le olvida moderar el tono de voz, pero estamos mejorando, hay motivación y compañerismo”.

"Los talleres nos permiten relajarnos y también ver cómo estamos, cómo estamos trabajando con los niños y con nuestra persona, es un momento donde podemos reflexionar lo que nos pasa y cómo superar los problemas con los niños /.../Tenemos que seguir realizándolos, reforzar el autocuidado y la comunicación”.

"Es importante el apoyo psicológico, estamos en constante desgaste no sólo en el trabajo también en la casa, una es el sustento de la familia y además de trabajar aquí llega a trabajar en la casa, y no se puede cambiar, necesitamos guí, que nos oriente”.

"Hay que seguir trabajando, sobretodo la coordinación en el equipo, la comunicación también hemos mejorado en la asertividad, pero todavía falta practicar más /.../ es importan- 
te tener más talleres donde se nos apoye cómo podemos intervenir con los niños, con sus problemas de conducta".

Al revisar los resultados cuantitativos, durante el año 2006, se observó un alto nivel de estrés psicosocial. Esto quiere decir que situaciones psicológicas y sociales del contexto en el cual se relaciona el personal del equipo, que trasciende a la situación laboral, estarían influyendo en su nivel de adaptación, rendimiento, cansancio y calidad de vida en el trabajo. El estrés psicosocial se relaciona con el cansancio emocional del equipo, lo que indica que habría que estudiar las causas o factores que estarían vinculando estas dos situaciones. Por otro lado, el alto nivel de realización profesional y satisfacción por la labor que realizan con los niños, niñas y jóvenes permite que el equipo no presente insensibilidad y despreocupación por la calidad de la atención en su trabajo.

Al comparar el estrés laboral de un año con otro que muestra el equipo, llama la atención que se presentan cambios mínimos, lo cual indica que habría que revisar las estrategias de autocuidado del equipo, indagar los factores que están relacionados con la situación de estrés, generar instancias de comunicación, discusión y reflexión para mejorar la calidad de la salud mental y física de las educadoras en el centro de protección y aprovechar el nivel de realización profesional para generar nuevas propuestas o cambios en la rutina y dinámica de trabajo que están ejecutando (Tabla 1 y 2 ).

Tabla 1. Comparación entre resultados 2005 y 2006 de factores de estrés laboral $(p<.05)$

\begin{tabular}{|c|c|c|c|c|c|c|c|c|c|}
\hline & & \multicolumn{5}{|c|}{ Diferencias relacionadas } & \multirow[t]{2}{*}{$\mathrm{t}$} & \multirow[t]{2}{*}{ gl } & \multirow{2}{*}{$\begin{array}{c}\text { Sig. } \\
\text { (bilateral) }\end{array}$} \\
\hline & & \multirow[t]{2}{*}{ Media } & \multirow[t]{2}{*}{ dt. } & \multirow[t]{2}{*}{$\begin{array}{l}\text { Error típ. de } \\
\text { la media }\end{array}$} & \multicolumn{2}{|c|}{$\begin{array}{l}95 \% \text { Intervalo de con- } \\
\text { fianza para la diferencia }\end{array}$} & & & \\
\hline & & & & & Inferior & Superior & & & \\
\hline Par 1 & $\begin{array}{l}\text { CE05 } \\
\text { CE06 }\end{array}$ & 4.30 & 39.621 & 12.529 & -24.04 & 32.64 & .343 & 9 & .739 \\
\hline Par 2 & $\begin{array}{l}\text { DP05 } \\
\text { DP06 }\end{array}$ & .10 & 44.660 & 14.123 & -31.85 & 32.05 & .007 & 9 & .995 \\
\hline Par 3 & $\begin{array}{l}\text { RP05 } \\
\text { RP06 }\end{array}$ & -4.00 & 38.410 & 12.146 & -31.48 & 23.48 & -.329 & 9 & .749 \\
\hline
\end{tabular}

En la aplicación previa no se analizó el estrés psicosocial que está más relacionado con el contexto personal y social de las participantes, pero se encontró necesario evaluar en la aplicación posterior al taller esta condición, con el fin de vincular otras variables que estuvieran influyendo en el autocuidado del personal. 
Tabla 2. Comparación de promedios de puntajes de estrés laboral

\begin{tabular}{llllcc}
\hline & & Media & $\mathrm{N}$ & $\mathrm{dt.}$ & Error típ. de la media \\
\hline Par 1 & CE05 & 55.70 & 10 & 24.882 & 7.868 \\
& CE06 & 51.40 & 10 & 31.263 & 9.886 \\
Par 2 & DP05 & 43.80 & 10 & 27.963 & 8.843 \\
& DP06 & 43.70 & 10 & 28.794 & 9.106 \\
Par 3 & RP05 & 58.10 & 10 & 16.822 & 5.320 \\
& RP06 & 62.10 & 10 & 30.127 & 9.527 \\
\hline
\end{tabular}

Se observó en el equipo un alto nivel de estrés psicosocial: esto quiere decir que situaciones psicológicas y sociales del contexto en el cual se relaciona el personal del equipo, que trasciende a la situación laboral, estarían influyendo en su nivel de adaptación, rendimiento, cansancio y calidad de vida. Al correlacionar el estrés psicosocial con las variables del estrés laboral, se observa lo siguiente (Tabla 3).

Tabla 3. Correlación entre estrés laboral y psicosocial. *: $p<.05 ; * *: p<.001 ; N=10$

\begin{tabular}{llcccc}
\hline & & CE & DP & RP & ESTRES \\
\hline CE & Correlación de Pearson & - & .139 & -.229 & $.799^{* *}$ \\
DP & Correlación de Pearson & .139 & - & $-.676^{*}$ & -.009 \\
RP & Correlación de Pearson & -.229 & $-.676^{*}$ & - & -.210 \\
ESTRÉS & Correlación de Pearson & $.799^{* *}$ & -.009 & -.210 & - \\
\hline
\end{tabular}

\section{Discusión}

Nos parece importante el poder compartir esta experiencia de intervención con educadoras de un hogar de menores, ya que puede resultar ilustrativa de la necesidad y a su vez de la viabilidad de desarrollar este tipo de trabajo de apoyo a la labor extenuante de las educadoras. De acuerdo a la evaluación obtenida se puede asumir con satisfacción el que se ha cumplido en buena medida con los objetivos planteados a partir del diagnóstico efectuado, lográndose llevar a cabo las actividades diseñadas para abordar las temáticas de relevancia identificadas por las educadoras para optimizar la calidad de la atención a los residentes del hogar. En dicho sentido se hizo efectiva la formación de conocimientos y su transferencia hacia las prácticas de rutina por parte de las educadoras. 
De este modo se puede observar que el modelo de buen trato propuesto por Barudy (2003) toma sentido, pues las educadoras logran generar instancias más asertivas y de mayor efectividad al trabajar desde un vínculo más afectivo en la resolución de conflicto con los beneficiarios, además de lograr modelar sus conductas, orientándolas hacia un mayor grado de adaptación a nivel psicológico y social generando un espacio de convivencia sano y constructivo. Entrevistas y observaciones realizadas con los niños, niñas, jóvenes y adultos del hogar, manifiestan que se sienten acogidos en el hogar, demostrando cariño y respeto por las educadoras, además de respetarse entre ellos y aprender a escucharse de una manera más comprensiva.

Sin embargo se considera importante potenciar más aún las estrategias de afrontamiento y autocuidado del personal del hogar. Los resultados nos muestran que el equipo está expuesto a un alto desgaste emocional en el contexto laboral, pero esto se ve amortiguado con la satisfacción que sienten por la labor en la atención de los menores, jóvenes y adultos. En este aspecto, Quintana (2005) y Morales, Pérez y Menares (2003) plantean que este desgaste emocional es característico de los grupos que trabajan con el sufrimiento humano, sin embargo plantean que es crucial que los equipos generen estrategias de autocuidado, supervición de sus procesos de intervención, autoevaluación, entre otros para fortalecer su intervención.

El alto nivel de realización profesional y satisfacción por la labor que realizan con los niños, niñas y jóvenes permite que el equipo no presente insensibilidad y despreocupación por la calidad de su trabajo y su atención.

Pero, el alto nivel de estrés se relaciona principalmente con otros contextos como es la familia y el entorno social de donde provienen las educadoras. Esto último se relaciona con el cansancio emocional en el trabajo, lo que nos indica sobre la importancia de intervenir y generar espacios de diálogo y comunicación de las situaciones personales, familiares y laborales que provocan ansiedad y angustia en el equipo, potenciando principalmente la acogida de todo el grupo, con la intención de apoyarse frente a las situaciones complejas tanto dentro como fuera del centro de atención (Morales, Pérez y Menares, 2003; Montgomery y Rupp, 2005; Quintana, 2005).

Se considera importante promover el autocuidado en el equipo con el fin de que las educadoras vivencien las intervenciones de una manera menos estresante, sean más efectivas 
y propositivas, y además presenten una mejor calidad de vida y salud mental en sus relaciones interpersonales tanto familiares, como personales y laborales.

Considerando lo anterior, se propone continuar con las capacitaciones profundizando los temas de comunicación personalizada y autocuidado para promover y mantener el buen trato, y generar instancias de comunicación de la experiencia entre el equipo. Además, se propone realizar una medición de los resultados relacionándolos con la calidad de vida, desarrollo y aprendizaje de los niños, niñas, jóvenes y adultos de dicho centro de atención, a la vez de realizar un seguimiento del impacto de los programas de capacitación en buen trato tanto en las educadoras como en los beneficiarios.

Como limitaciones del estudio, es importante tener presente las experiencias y edades tan diversas del equipo profesional, lo que hace que los resultados tengan una alta desviación desde la media, y en este sentido se observa que las mujeres más jóvenes tienden a presentar mayor estado de estrés que las con más experiencias. Si se observa sus historias personales, la mayoría de ellas es jefa de hogar, por lo tanto habría que indagar más de manera cualitativa sus experiencias de vida y desde ahí proponer estrategias de intervención para poder mejorar la calidad de vida de las educadoras. Otro aspecto importante ha considerar es que los resultados sólo representan a las educadoras de trato directo, y no a los equipos profesionales y otros funcionarios encargados del aseo, alimentación y locomoción. Sería importante tener la visión y experiencia de ellos como para configurar de una manera mucho más completa la intervención y guía en la convivencia y buen trato de todos los participantes del centro de atención de menores descrito. 


\section{Referencias}

Aracena, M., Castillo, R., Haz, A., Cumsille, F., Muñoz, S., Bustos, L. y Román, F. (2000). Revista de Psicología Universidad de Chile, 9, 1 - 21.

Arón, M. y Milicic, N. (1993). Vivir con otros. Santiago: Universitaria.

Barudy, J. (2003). Promover el buen trato y los recursos resilientes como bases de la prevención tratamiento de las consecuencias de la violencia humana. Consultado el 20 de marzo 2006 en: http://www.peretarres.org/eutses/recursos/inauguracio_vio.pdf.

Buzzetti, M. (2005) Validación del Maslach Burnout Inventory (MBI), en dirigentes del colegio de profesores de Chile. Memoria para optar al Título de Psicólogo, Universidad de Chile.

De la Fuente, J., Peralta, F.J. y Sánchez, M.D. (2006). Valores sociopersonales y problemas de convivencia en la educación secundaria. Electronic Journal of Research in Educational Psychology, 4(2), 171-200. (www.investigacion-psicopedagogica.org/revista)

Domínguez, R. y Narváez P. (2004). Violencia y salud mental. Santiago: Unidad de Salud Mental, Ministerio de Salud.

Holmes, T. y Rahe, R. (1967). Escala de estrés psicosocial. Consultado el 23 de marzo de 2006 en:

http://www.uam.es/departamentos/medicina/psiquiatria/comunicologia/tema2.html

Justicia, F. Benítez, J.L., Fernández, E. Pichardo, M.C., García-Berbén, T. y Fernández, M. (2006). Aproximación a un nuevo modelo explicativo del comportamiento antisocial. Electronic Journal of Research in Educational Psychology, 4(2), 131-150.

Magendzo, A., Donoso, P. y Rodas, M. (1997). Los objetivos fundamentales transversales en la reforma educacional chilena universitaria. Santiago Chile: Editorial Universitaria.

Marty, C y Carvajal, C. (2005). Maltrato infantil como factor de riesgo de trastorno por estrés postraumático en la adultez. Revista Chilena de Neuropsiquiatría, 43 (3), 180 - 187.

Maslach, C. y Jackson, S. (1997). MBI. Inventario Burnout de Maslach. Síndrome del "quemado" por estrés laboral asistencial. Madrid: TEA

Maturana, H. y Verden - Zöller, G. (2000). Amor y juego. Fundamentos olvidados de lo humano. Santiago: Dolmen.

MINEDUC (2004). Convivencia escolar: metodologías de trabajo para escuelas y liceos. República de Chile: Ministerio de Educación.

MINSAL (1999). Relaciones saludables en ambiente pre-escolar. República de Chile: Ministerio de Salud. 
Montgomery, C. y Rupp, A. (2005). A meta-analysis for exploring the diverse causes and effects of stress in teachers. Canadian Journal of Education, 28 (3), 458 - 486.

Montes, C. y Montoya, G. (2003). Guía para adultos campaña del buen trato: Estrategias para fomentar el buen trato en adultos, niños, niñas y adolescentes. Consultado el 20 de marzo de 2006 en: http://www.scslat.org/search/publi.php?_cod 73 lang_s..

Morales, G., Pérez, C. y Menares, M. (2003). Procesos emocionales de cuidado y riesgo en profesionales que trabajan con el sufrimiento humano. Revista de Psicología de la Universidad de Chile, 12 (1) 9 - 25.

Quintana, C. (2005). El síndrome de Burnout en operadores y equipos de trabajo en maltrato infantil grave. Psykhé, 14 (1), 55 - 68.

Rivera, A; Vollmer, P; Aravena, R; Carmona, A. (1985) Escala de evaluación del reajuste social de Holmes y Rahe: validación para una población de estudiantes y empleados chilenos; estudio piloto. Revista de Psiquiatría Clínica, 22, 113-123.

Robaina, G. (2001). El maltrato infantil. Revista Cubana de Medicina General, 17 (1), 74 - 80

Rogers, C. (2000). El proceso de convertirse en persona. Mi técnica terapéutica. Madrid: Paidós.

Servicio Nacional de Menores SENAME (2006). Línea de Protección, Normas Técnicas de Funcionamiento e Intervención en los Centros de Protección Simple. Santiago de Chile.

Vizcarra, M., Cortés, J., Bustos, L., Alarcón, M. y Muñoz, S. (2001). Maltrato infantil en la ciudad de Temuco. Estudio de prevalencia y factores asociados. Revista médica de Chile, 129 (12), $1425-1432$. 